\title{
The Conceptual Framework of Ubiquitous Computing for Home Energy Monitoring by Using Interactive Interface Design
}

\author{
Tapanee Treeratanaporn
}

\begin{abstract}
Ubiquitous computing is about bringing computing to people's physical world, integrating with objects and activities. In this paper, ubiquitous computing is applied for energy monitoring. Currently, energy saving becomes the most significant issue in our world. We apply the principles of HCI (Human Computer Interaction) to design user interface because the main important variable for energy saving is human behavior as well. Interactive interface including both visualization and sound are proposed for the usability's goal in this system. The displays show energy usage in each electric appliance in the house all the time. We believe that this framework can strongly induce intrinsic and extrinsic motivation for energy-aware consumption indeed.
\end{abstract}

Keywords-Energy monitoring, Ubiquitous computing, Human computer interaction

\section{INTRODUCTION}

$\mathrm{E}$ NERGY saving is one of the most current significant issues that our globe concerned. Every country around the world must be responsible to reduce and control energy consumption. In the previous studies, many projects can certainly provide whole-house energy consumption information in the house to consumers such as AWARE Clock project, Digital Home Energy Management System (Dehems), AlertMe, and Current Cost. Most of them are the good tools that provide energy consumption [1]. However, they presented information in the term of aggregate format, by showing a single value for the total consumption over past days or past weeks so the consumers cannot know their behaviors clearly.

Electric appliances are enormously various. Fig. 1 shows average power (watts) consumption in each electric appliance. It is very important that we should reasonably realized for home environment to monitor and control the uses of energy for the households such as lighting, air conditioning, refrigerator, microwave or electric oven, washing machine, television, coffee maker, iron, and so on.

Tapanee Treeratanaporn is with the Electrical and Computer Engineering Department, King Mongkut's University of Technology North Bangkok, Bangkok, Thailand.

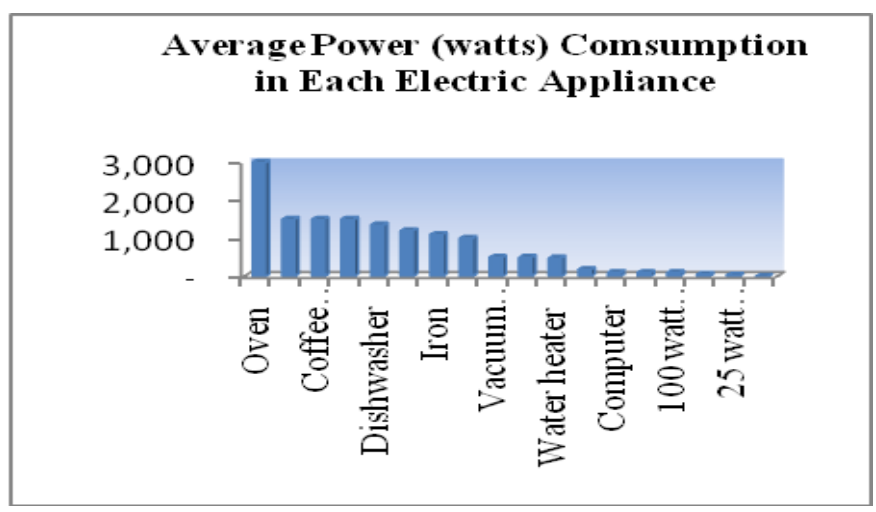

Fig. 1 Average power (watts) consumption in each electric appliance

Many studies of energy identify that one of the significant problems in energy saving is human behaviors [2]. The studies of energy displays identify that main problems of current designs is the lack of context around the information provided and the consequent difficulties that users encounter in making sense of this information [3-5]. If we can present the display or feedback to consumers concerning their energy usage effectively in each electrical appliance, we believe that it can induce intrinsic motivation for energy-aware consumption to consumers.

Therefore, in this paper we propose the framework that considerable interest over the last few years in ubiquitous computing to motivate the energy saving and Human Computer Interaction (HCI) to effectively display for energy consumption.

\section{LITERATURE REVIEW}

\section{A. Ubiquitous Computing}

Mark Weiser, the founder of Ubiquitous Computing, defined that "Ubiquitous computing enhances computer use by making many computers available throughout the physical environment, but making them effectively invisible to the user. It is about bringing computing to people's physical world, integrating with everyday objects and activities [6].

Ubiquitous computing is considered as a path of innovation, which refers to a computing scheme containing various computing devices or information appliances embodied in the environment [7]. Applying of ubiquitous computing is currently in the business, public and private sectors such as in energy management, trade, logistics, industry, transport, healthcare, as well as personal everyday life [8]-[11]. 
The main components of ubiquitous computing infrastructure consist of sensor network, data communication, databases or data storage and user application in each device.

Sensor is one of the most important components in ubiquitous computing. A sensor network consist of a large number of tiny autonomous computing devices, each equipped with sensor: a wireless radio, a processor, and a power source.

Sensors that used in the energy monitoring systems can be divided into two types. These are referred to as non-intrusive and intrusive sensor.

For non-intrusive systems consist of only a single sensor which is deployed for the whole household [12] as shown in Fig. 2. The energy sensor sends the whole energy consumption to the power meter.

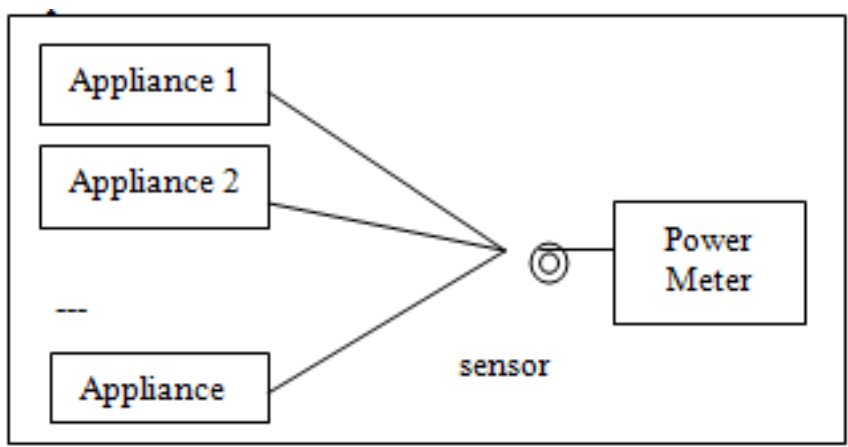

Fig. 2 Non-intrusive energy monitoring

The non-intrusive sensor can be placed at the main terminal where all the power lines are combined (such as a distribution box).

On the other hand, intrusive sensors are installed at every appliance since a multiple number of sensor devices are required to be attached to each appliances [12] as shown in Fig.3. The energy sensors are attached to each appliance. They can send electricity usage in individual appliances.

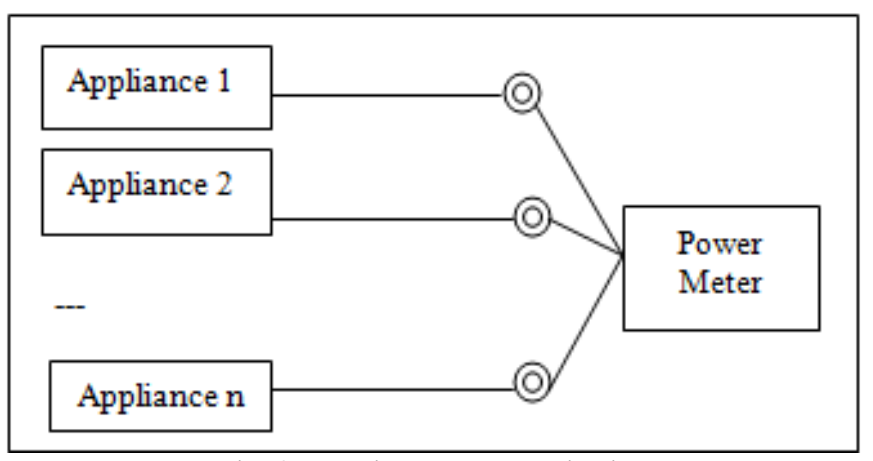

Fig. 3 Intrusive energy monitoring

However, many studies showed that the good energy consumption information to home consumers should engage with and understand their consumption data, relating it to concrete activities in their life. Users should encounter in making sense of this information [13]. Hence, energy consumption in individual appliances such as air conditioners, hot water heaters, dishwashers, refrigerators, electric vehicles, and other loads should separately display.

We can apply a new automatic method for the disaggregation of appliance loads from whole-house metering is, so called NILM (Non- Intrusive Load Monitoring) as well. NILM is a single-point sensing that reports household-level energy related activities. This technique involves machine learning and optimization algorithms. It can breakdown energy consumption at appliance level and make cost reduction [12].

\section{B. Human Computer Interaction}

Human is one of the main problems in energy saving. Users should engage and understand their energy consumption data, relating in each activities in everyday life. Hence Human Computer Interaction (HCI) and user interface design that concerned with human and their activities should be strongly considered. The three important aspects in HCI consist of human, computer and interaction.

Human factor (user) is the art and science of understanding the properties of human capability. Everything that is sensed (sight, hearing, touch, smell, taste) are considered to be information which the mind processes. Human cognition is what is going on in our heads when we carry out our everyday activities.

Computer factors affect the interaction including input devices e.g. text entry or pointing, output devices e.g. screen (small or large), physical interaction e.g. sound, haptics, bio-sensing, memory, RAM, permanent media, capacity as well as access processing.

In the last component, interaction is how you go about doing it or ultimately in terms of operations or actions. Common interaction styles in a computer consist of command line interface, menus, natural language, question/answer and query dialogue, form-fills and spreadsheets, WIMP, point and click, and three-dimensional interfaces.

\section{CONCEPTUAL ARCHITECTURE FRAMEWORK}

Conceptual architecture framework proposal in this paper consists of three main parts: 1) Data Collection, 2) Database, and 3) Application..

1) Data Collection: Data collection is a physical level configuration that consists of sensors. Sensors measure and send energy usage data in each electric appliance to database for storing and analyzing.

2) Database and computation: Database and computation unit comprises of three modules: a database, a machine learning unit, and a web data crawler. Database stores all energy usage. Machine learning is responsible for processing, analyzing, and calculating the information. It consists of event detection, machine learning, and logical reasoning algorithms. The main functions are to calculate the pricing and compare energy usage in the past time and the current time. The last module is web data crawler. It consists of a set of software programs that retrieves energy tariff and pricing rate from web repository.

3) Application: Application services can provide energy usage information, usage comparisons based on historical data, and price feedback to a consumer in each electric appliance on a real time. The feedback can display through a web interface, which can also be configured on an in-home portable display or smart phone. 
Fig. 4 shows the conceptual architecture framework of ubiquitous computing for home energy monitoring by using interactive interface design. The framework consisting of three main components: 1) data collection, 2) database, and 3) application.

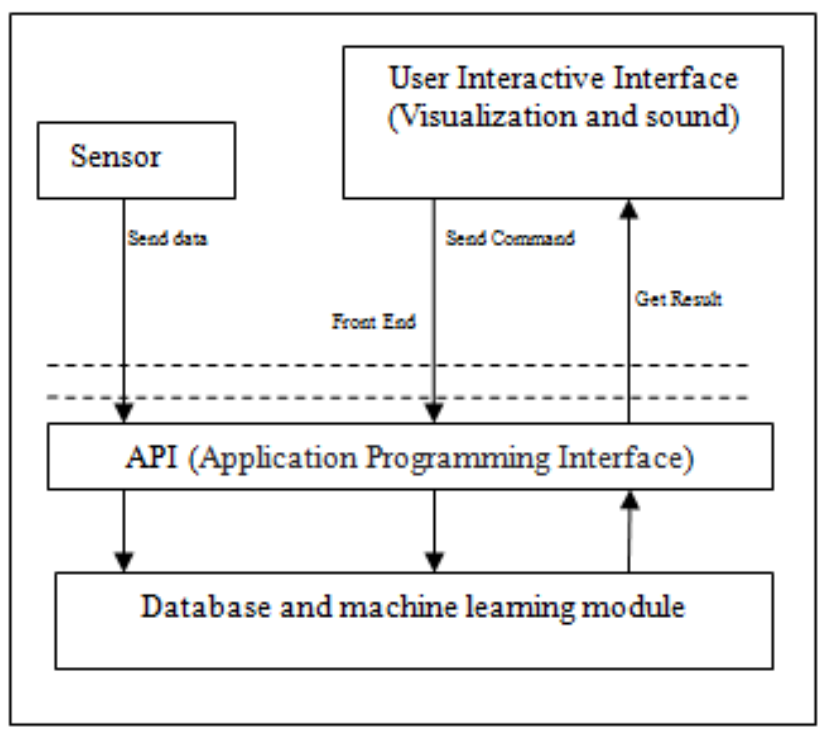

Fig. 4 Conceptual architecture framework of ubiquitous computing for home energy monitoring by using interactive interface design

Although many projects can certainly provide both whole or disaggregated energy consumption information in each electric appliance to home consumers, some studies of energy management point out that one of the main problems of energy consumption information is displaying or user interface design [3]-[4]. Hence, effective display or user interaction interface for energy consumption is very important that we should strongly realize.

\section{USER INTERFACE DESIGN}

The interface design must balance both technical functionalities as well as visual element design. The principles of user interface design for this paper are 1) To keep the interface simple. The best interfaces are almost invisible to the user and clear in the language that are used on labels and in messaging and 2) To make sure that the system communicates what's happening such as inform users when changes in state, or errors. The use of various user interface elements to communicate status is a good way [13]. Providing feedback, happening, and a notification are update messages that announced something new for the users to know. [14]-[15].

Feedback is an important tool for user interface design. Many academic and commercial projects stated that visualization of electricity consumption is a good tool for display. [14]-[15]. However, we think that the use of various user interface elements to communicate status such as sound feedback is a better way to establishing energy saving motivation.

Sound feedback is a feature to help users know on their activities without needing to look at the screen. It works through a speaker device that normally installed in computer or smart phone. When users perceive various senses: both sight and hearing, it can motivate users to change behavior.

\section{RESULT}

When users used each electric appliance, a sensor measured and sent energy usage data to database via API. Database that consists of event detection, machine learning, and logical reasoning algorithms module collected energy usage and calculated the pricing that retrieves energy tariff and pricing rate from web repository to mobile. Finally it can compare energy usage and price between the recent and the past time.

User can know energy usage in each appliance and time, (s)he can command on mobile application. The good interactive interface design has many displays that user can select by themselves following to the context and situation that wanted.

When we adopt this ubiquitous application for energy monitoring, it can show energy usage in each electric appliance all the time via interactive interface both visualization and sound.

Examples of main function displays are 1) menu that created and listed the electrical machines in the system 2) menu that showed the feedback when using energy usage exceeds and 3) menu that controlled the usage setting as Fig. 5, 6, and 7 shown below respectively.

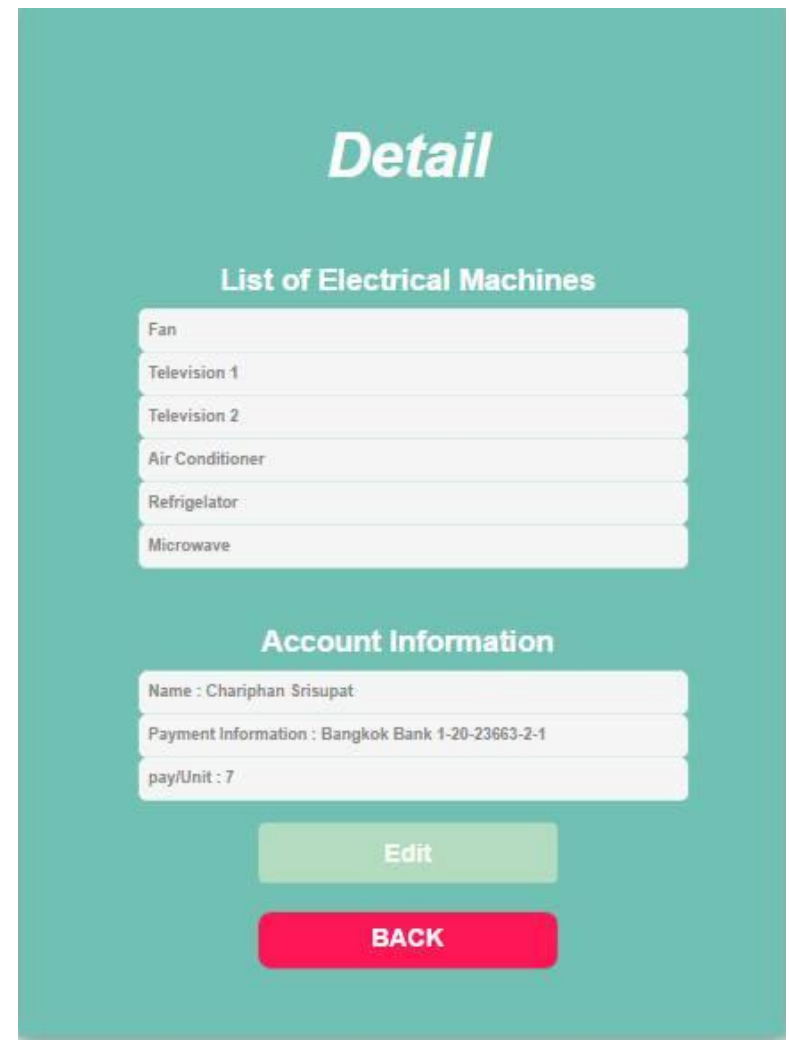

Fig.5 Menu that created and listed the electrical machines in the system 


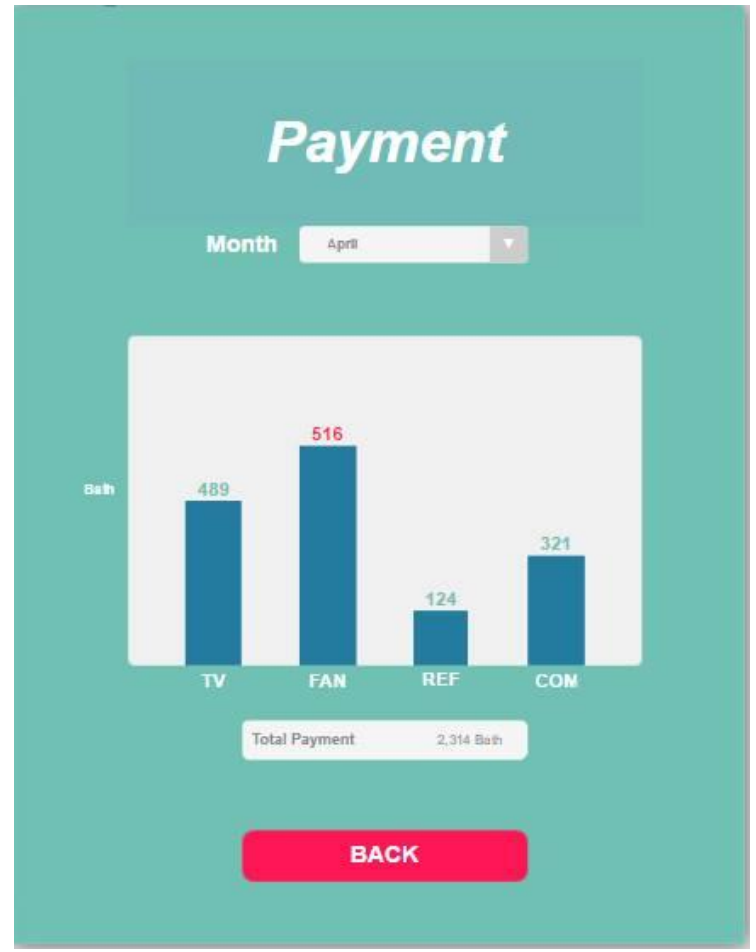

Fig. 6 Menu that showed the feedback when using energy usage exceeds

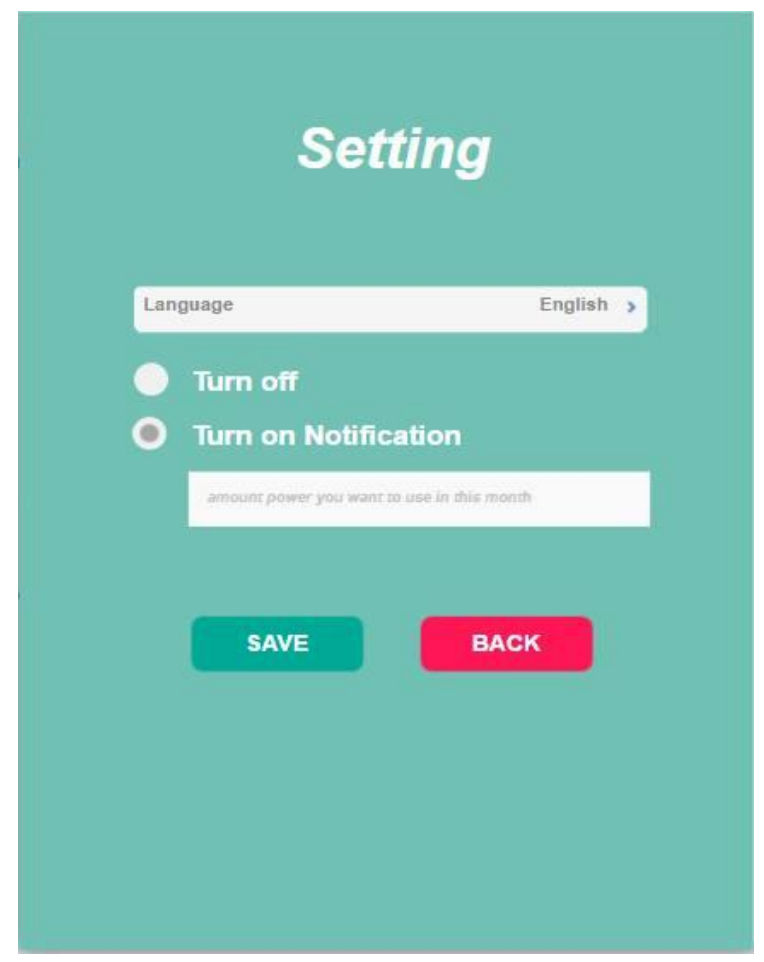

Fig.7 Menu that controlled the usage setting

We gave 30 participants used the home energy monitoring interactive interfaces. The results showed that most of them (86\%) were strongly satisfied and believed that this framework can really induce motivation for energy-aware consumption in everyday life because users can know energy usage in each appliance. Furthermore, they can select and control the context and situation that they wanted.

\section{ACKNOWLEDGMENT}

The author is thankful KMUTNB forth year electrical and computer engineering undergraduate students: Chariphan Srisupat, Sirinakorn Soonthornsarn, and Veerachai Phu Sam Lee who designed and developed user interfaces for this project.

\section{REFERENCES}

[1] S. Ramchurn, P. Vytelingum, and A. Rogers, "Putting the "smarts" into the smart grid: A grand challenge for artificial intelligence". The proceedings of CACM, 2011.

[2] S. Fischer, "Feedback on household electricity consumption: a tool for saving energy". Energy Efficiency 1, 1, 2008, 79-104.

[3] G. Fitzpatrick, and G. Smith, "Technology- enabled feedback on domestic energy consumption: Articulating a set of design concerns". IEEE Pervasive Computing. January, 2009, 37-44.

http://dx.doi.org/10.1109/MPRV.2009.17

[4] Y. Strengers, "Designing eco-feedback systems for everyday life," The proceedings of CHI '11, ACM, 2011, 2135-2144.

[5] M. Friedewald and O. Raabe, "Ubiquitous computing: an overview of technology impacts," Telematics and Infomatics, 2011.

[6] J. Krumm, "Ubiquitous advertising: the killer application for the 21st century". IEEE Pervasive Computing, 2011. http://dx.doi.org/10.1109/MPRV.2010.21

[7] D. Comes, C. Evers, and K. Geihs, "Designing Socio-technical Applications for Ubiquitous Computing". Distributed Applications and Interoperable Systems, 2012.

http://dx.doi.org/10.1007/978-3-642-30823-9_17

[8] S. Dian Shen, L. Junzhou, and D. Fang, "Energy-efficient resource allocation model with QoS assurance for ubiquitous and heterogeneous environment," the proceedings of 7th International Conference on Ubi-Media Computing and Workshops, 2014.

[9] V. Sundramoorthy, G. Cooper, N. Linge, and Q. Liu, "Domesticating energy-monitoring systems: Challenges and design concerns". IEEE Pervasive Computing, 2011, 20-32.

http://dx.doi.org/10.1109/MPRV.2010.73

[10] G. Fitzpatrick and G. Smith, "Technology- enabled feedback on domestic energy consumption: articulating a set of design concerns",", IEEE Pervasive Computing, 2009, 37-44. http://dx.doi.org/10.1109/MPRV.2009.17

[11] Y.A.Strengers, "Designing eco-feedback systems for everyday life. The proceedings of CHI '11, ACM 2011, 2135-2144.

[12] M. Zeifman and K. Roth, "Nonintrusive appliance load monitoring: review and outlook. Consumer Electronics", IEEE Transactions. 2011, 57,1, 76-84.

[13] A.H. Kazmi, M.J. O'Grady, and G.P. O'Hare, "Energy management in the smart home". The proceedings of ACM UbiComp, 2012.

[14] E. Costanza, D.S. Ramchurn, and N.R. Jennings, "Understanding domestic energy consumption through interactive visualisation: a field Study", The proceedings of IEEE 10th International Conference on Ubiquitous Intelligence, 2013.

[15]V. Sundramoorthy, G. Cooper, N. Linge, and Q. Liu, "Domesticating energy-monitoring systems: Challenges and design concerns". IEEE Pervasive Computing, vol. 10, no. 1, 2011, 20-27.

http://dx.doi.org/10.1109/MPRV.2010.73 